\title{
ANFIS-based Wireless Sensor Network (WSN) Applications for Air Conditioner Control
}

\author{
Yi-Jen Mon ${ }^{1}$, Chih-Min Lin ${ }^{2}$, Imre J. Rudas ${ }^{3}$ \\ 1 Department of Computer Science and Information Engineering, Taoyuan Inno- \\ vation Institute of Technology, Chung-Li, Taoyuan, 320, Taiwan \\ e-mail: monbuy@tiit.edu.tw \\ 2 Department of Electrical Engineering, Yuan Ze University, Chung-Li, Taoyuan, \\ 320, Taiwan, e-mail: cml@saturn.yzu.edu.tw \\ 3 Óbuda University, Bécsi út 96/B, H-1034 Budapest, Hungary \\ e-mail: rudas@uni-obuda.hu
}

\begin{abstract}
The adaptive network fuzzy inference system (ANFIS)-based wireless sensor network (WSN) is designed to serve as a monitor of controller of an indoor air-conditioning system. The WSN comprises sensors to monitor the temperature of the indoor space and the ANFIS controller is used to control the fans in order to obtain energy-saving benefits. By using the application programming interface (API) of WSNs, many applications have been developed. The experimental results demonstrate that good data transference and control performance have been achieved.
\end{abstract}

Keywords: wireless sensor network; ANFIS; air conditioner control

\section{Introduction}

In this paper, an adaptive network fuzzy inference system (ANFIS)-based wireless sensor network (WSN) control system is developed. A reduction in energy consumption is the main challenge of the WSN [1]. The IEEE 802.15.4/ZigBee standard has been proposed by the ZigBee Alliance to develop standards for costeffective and low-power consumption WSNs [2-10]. By using the lower cost, lower power consumption ZigBee WSN and efficient ANFIS controller, many control applications can be achieved through the network. Recently, many applications of indoor, outdoor and mobile devices, such as home automation and security, vehicle space automation, consumer products, health-care, environmental monitoring and indoor location identification, etc., have been developed to improve human quality of life. In particular, these WSNs can provide ideal networking solutions for lower cost and simple installations $[1,2]$. 
The ZigBee, which builds upon the IEEE 802.15.4 standard, defines the physical layers and media access control (MAC) layers for lower cost, lower rate, personal area networks (PAN). There are three types of ZigBee network topologies defined. These networks topologies are star, tree and mesh network topologies, which are provided by a framework of application programming interfaces (API) in the application layer [2-10].

The ANFIS [11] was proposed many years ago and is widely used in research works. The ANFIS reveals an efficient learning network and its applications can be found in many works in the literature [12-14]. In this paper, the ANFIS controller is used to control the motors of fans for indoor temperature control. The ANFIS controller is a user-friendly algorithm and serves as a method for inducing many fuzzy if-then rules with suitable membership functions to generate the relationship fuzzy associated memory (FAM) pairs of inputs/outputs (I/O) and reasonable fuzzy rules so as to achieve a fuzzy inference system (FIS). A reliable controller can be designed based on this FIS. MATLAB ${ }^{\mathrm{TM}}$ Ltd. has provided very useful and user-friendly tools for engineers to design this ANFIS controller [15].

In the solution reported in this paper, by using the data measured via the WSN, data transference and the fan's motor control are performed by the proposed ANFISbased WSN control methodology. This method has great benefits, such as reducing the power consumption of the indoor space, improving the efficiency of airconditioning and saving energy.

\section{Preliminary of ZigBee WSN}

Regarding the MAC layer of ZigBee and integrated peripherals of the WSN microcontroller (WMCU), the Application Queue APIs (AQA) of the WSN microcontroller are provided a queue-based interface between applications and both the ZigBee stack and the hardware drivers. These AQAs are used to deal with many interrupts coming from the MAC layers. There are three types of interrupt implemented by using APIs. The first type is used for the MAC data services, the second type is used for the MAC management services and the third type is used for handling interrupts of hardware drivers $[16,17]$.

The basic type of network topology is the star topology, which comprises a central personal area network (PAN), which is called 'coordinator', surrounded by the other nodes of the network, which are called 'end device'. The tree network topology has an implicit structure based on parent-child relationships. In the mesh network topology, all devices can communicate directly and can be identical in an adhoc network $[16,17]$. In this paper, the star topology of a WSN is used to develop the air conditioner controller. Data transference between network nodes can be searched or found by any request. The data transference methods are shown in 
Fig. 1. When transferring data from a coordinator to a node, the node might not always be ready to receive the data. In this case, the node will be requested to receive data frame. Afterwards, the 'Acknowledgment' message is sent from coordinator. Finally, the end device will send the 'Acknowledgment' message to the coordinator once the data transferring has finished. [16, 17].

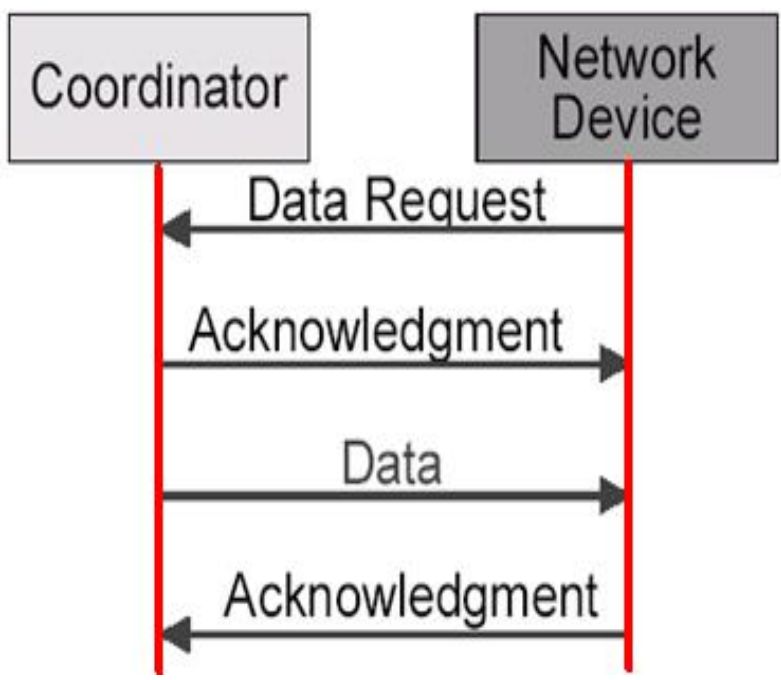

Figure 1

The diagram of data transference method of a WSN

\section{ANFIS-based WSN Controller Design}

The algorithm of ANFIS is based on a least-squares estimation (LSE) and back propagation gradient descent methods to identify the membership functions' parameters so as to achieve fuzzy inference systems (FIS). A neural network structure of ANFIS is shown in Fig. 2. This methodology requires a pair of input/output data to train the FIS membership function parameters. The developed method of ANFIS is briefly described as follows [11]:

$$
\begin{array}{r}
R_{i}: \text { If } x_{1} \text { is } A_{i 1} \quad \text {...and } x_{n} \text { is } A_{\text {in }} \\
\text { then } u_{i}=p_{i 1} x_{1}+. .+p_{i n} x_{n}+r_{i}
\end{array}
$$

where $R_{i}$ denotes the $i$ th fuzzy rules, $i=1,2, . ., r ; A_{i k}$ is the fuzzy set in the antecedent part associated with the $k$ th input variable at the $i$ th fuzzy rule, and $p_{i 1}, \ldots, p_{i n}, r_{i}$ are the fuzzy consequent parameters. 
The defuzzification of the output $u$ can be calculated by the method of averaged weight as follows:

$$
\begin{aligned}
u & =\frac{w_{1}}{w_{1}+. .+w_{n}} u_{1}+. .+\frac{w_{n}}{w_{1}+. .+w_{n}} u_{n} \\
& =\bar{w}_{1} u_{1}+. .+\bar{w}_{2} u_{n}
\end{aligned}
$$

where $w_{i}$ is the $i$ th node output firing strength of the $i$ th rule, and $\bar{w}_{1}=\frac{w_{1}}{w_{1}+. .+w_{n}}, . ., \bar{w}_{n}=\frac{w_{n}}{w_{1}+. .+w_{n}}$.

Based on the reason of Takagi-Sugeno (T-S) type fuzzy inference system, the output of ANFIS can be calculated as $u_{i}=p_{i 1} x_{1}+. .+p_{i n} x_{n}+r_{i}$, then Eq. (2) can be expressed as

$$
\begin{aligned}
u & =\bar{w}_{1} u_{1}+. .+\bar{w}_{n} u_{n} \\
& =\left(\bar{w}_{1} x_{1}\right) p_{i 1}+. .+\left(\bar{w}_{1} x_{n}\right) p_{i n}+\left(\bar{w}_{1}\right) r_{1} \\
& + \\
& \vdots \\
& +\left(\bar{w}_{n} x_{1}\right) p_{i 1}+. .+\left(\bar{w}_{n} x_{n}\right) p_{i n}+\left(\bar{w}_{n}\right) r_{n} .
\end{aligned}
$$

The ANFIS's algorithm can be applied directly to Eq. (3) [11]. In the forward direction of the ANFIS algorithm, input signals go forward until layer 4 of Fig. 2 and the consequent parameters $p_{i 1}, p_{i 2}, r_{i}$ are changed. In the backward direction, the error values are feedbacked and used to update the premise parameters $x_{1}, x_{2}$. When all the values of the parameters are changed, the membership functions are also modified; thus every membership functions of $A_{i 1}$ and $A_{i 2}$ are induced.

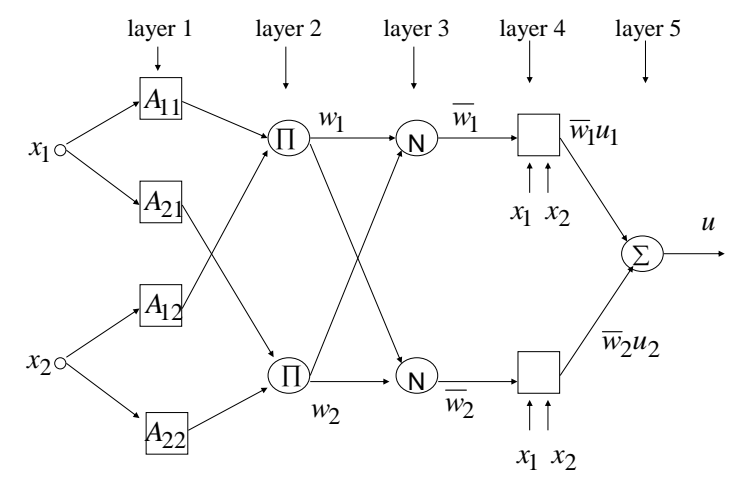

Figure 2

A two-input one-output ANFIS architecture 


\section{Experimental Results}

The program of the WSN is developed on free software called Code::Blocks. First, the program of the coordinator is developed and then the program of the end device is developed. Every network must have one and only one PAN coordinator, and one of the tasks in setting up a network is to select and initialise this coordinator. The network setup process is shown in Fig. 3. The main program of the coordinator and the end device are developed in $\mathrm{C}$ language. The architecture of the coordinator software is shown in Fig. 4, especially the API of 'vProcessEventQueues', which is the most important subroutine of this system. For the configuration program, the personal area network identification (PAN-ID) of every end device must be set adequately. The development board is produced by Fontal Technology Inc., Taiwan. This is a high-power ZigBee Kit (named FT-6200). It can provide all the software tools and hardware required to obtain first-hand experience with the WSN. The entry-level kits contain one base development board (BDB) and one sensor development board (SDB). Each board is equipped with a high-power IEEE 802.15.4/ZigBee RF module based on the JN-5121 WMCU, which provides a much higher coverage range with a $2.4 \mathrm{GHz}$ RF antenna, which has the IPEX connector for easier mechanical design than the normal power RF module. For the I/O expansion ports, it has 10 useful pins of general purpose input/output (GPIO), which include the universal asynchronous receiver/transmitter (UART), analogue-to-digital converter (ADC), digital-to-analogue converter (DAC) and Comparator. The sensor development board features temperature and humidity sensors $[16,17]$. The development board is shown in Fig. 5.

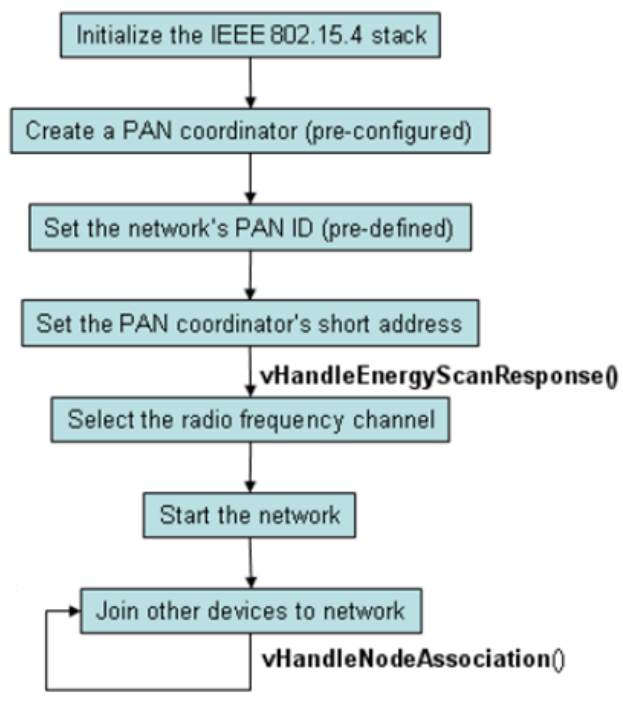

Figure 3

Diagram of network setup process 


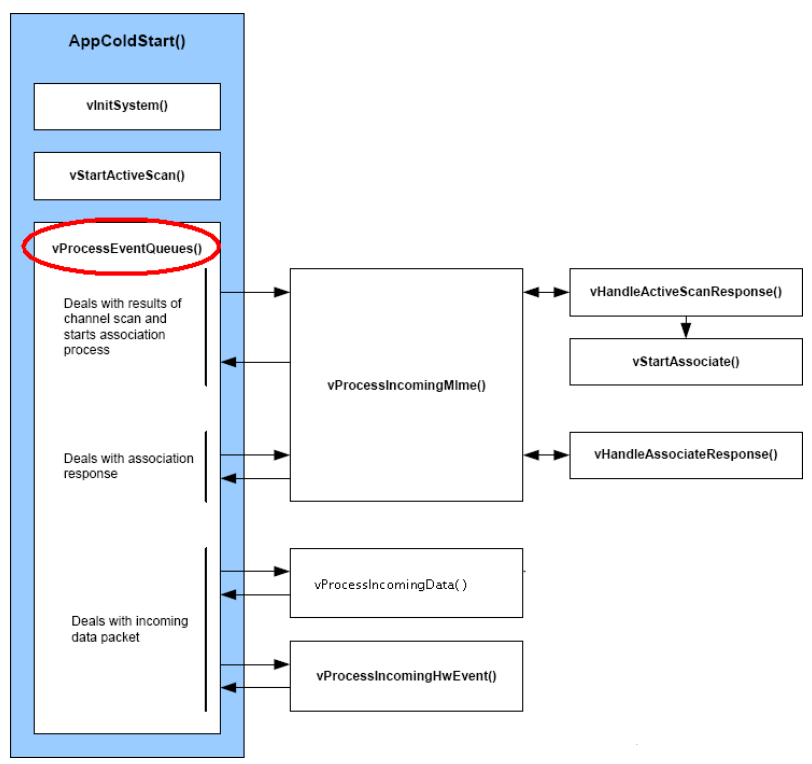

Figure 4

Diagram of coordinator software architecture of WSN

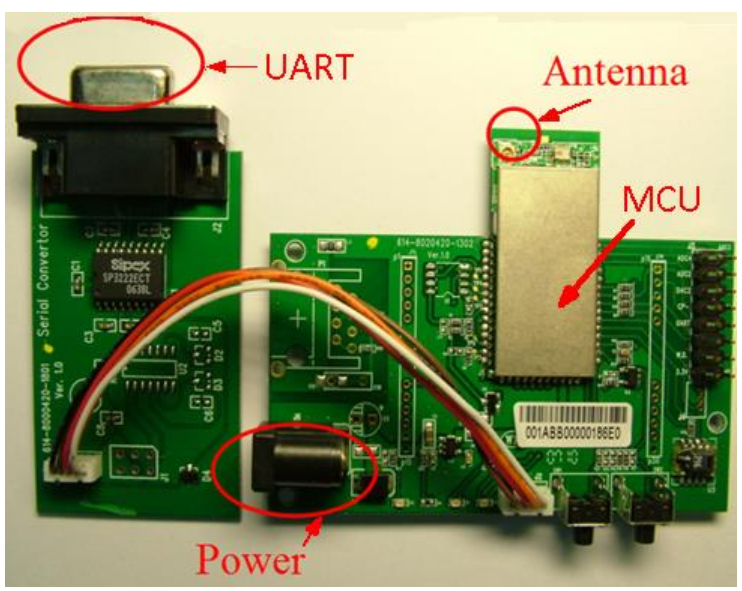

Figure 5

Development boards of WSN

For the software, Jennic Technology Inc. also provides free Application Programming Interface (API) packages to the peripheral devices on the JN5121 and JN513x single-chip IEEE 802.15.4/ZigBee compliant wireless microcontrollers. This is known as the Integrated Peripherals API. It details the calls that might be made through the API in order to set up, control and respond to events generated by the peripheral blocks, such as the UART, GPIO lines and Timers, among others. 
The software invoked by this API is present in the on-chip ROM. This API does not include support for the ZigBee WSN MAC hardware built into the device; this hardware is controlled using the MAC software stack that is built into the on-chip ROM [16, 17].

ZigBee can be used with different sensors, such as: in-vehicle or home automation, security management, industrial, environmental controls and personal medical care. In this paper, the ZigBee WSNs are used to design the indoor air conditioner controller by means of the ANFIS-based WSN control methodology. The design concept diagram of the ANFIS-based WSN control is shown in Fig. 6. A star topology network is used in this paper. By using UART, the data can be displayed in the LCD of different end devices sensors located in four corners of the indoor space. Monitoring of the temperature is one of the main experimental aims; the temperature sensors on the end devices transmit data to the coordinator, which are then also displayed in the LCD through UART. An actual implementation of the air conditioner control in the laboratory is shown in Fig. 7. By simulation, the ANFIS-based controller described in section 3 is developed. Four end devices are used to measure temperature and then transmit that data to the coordinator. After manipulation by the ANFIS-based WSN controller, the fuzzy values are returned to the end devices located in the four corners of the indoor spaces to control the fans and adjust the temperature. The results of the ANFIS design are shown in Fig. 8. Especially from Fig. 8(b) of this simulation, reasonable fan speeds normalized from 0 to 1 are achieved. In this condition, for example, WSN1 has the highest temperature; thus, fan1 will be speeded up the most because it has the highest fuzzy number of 0.941. In this experiment, the GPIO of every end devices is used to perform the digital-to-analogue (D/A) transformation to send the signal to drive the motor of the fan. The results of the ten-hour continuous simulation are presented in Fig. 9. It can be clearly verified that fan 1 will be fully active from the $4^{\text {th }}$ hour to the $5^{\text {th }}$ hour, as shown in Fig. 9(b). From these empirical tests and simulations, the WSN control of temperature monitoring is successfully established and good performance of the fan motor control is achieved.

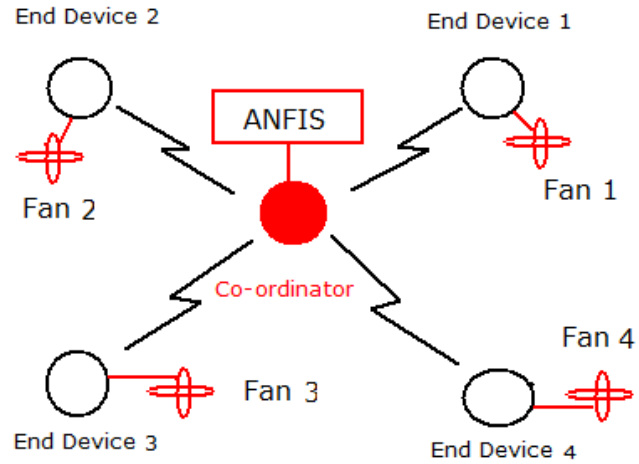

Figure 6

Design concept diagram of ANFIS-based WSN for air conditioner control 


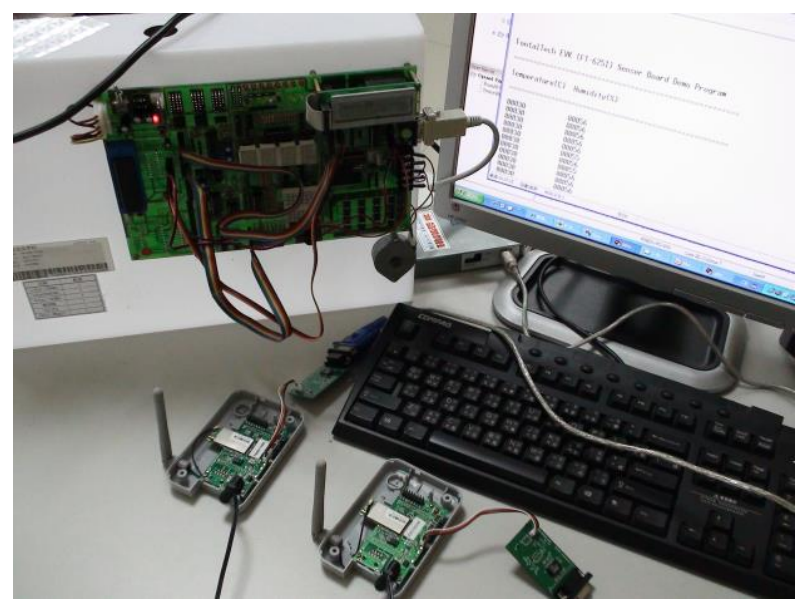

Figure 7

Implementation diagram of WSN for indoor temperature monitor

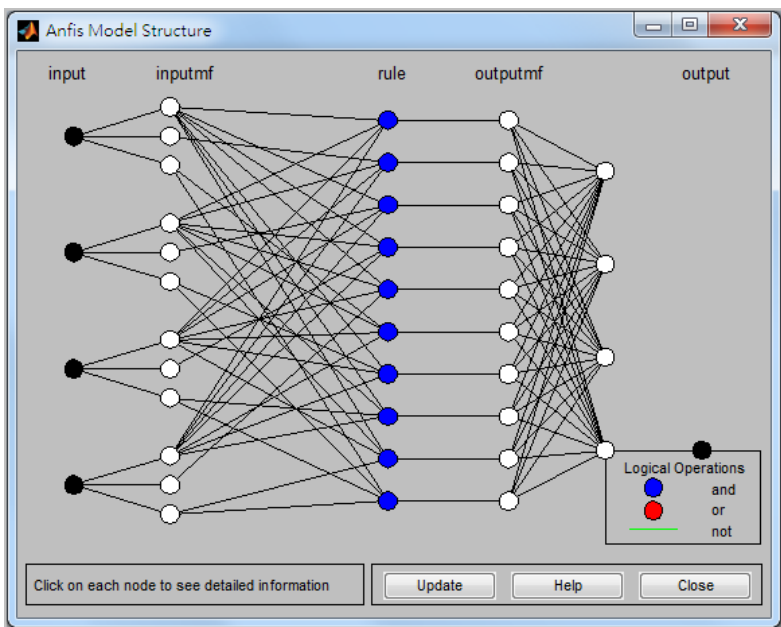

Figure 8 (a)

ANFIS structure diagram 


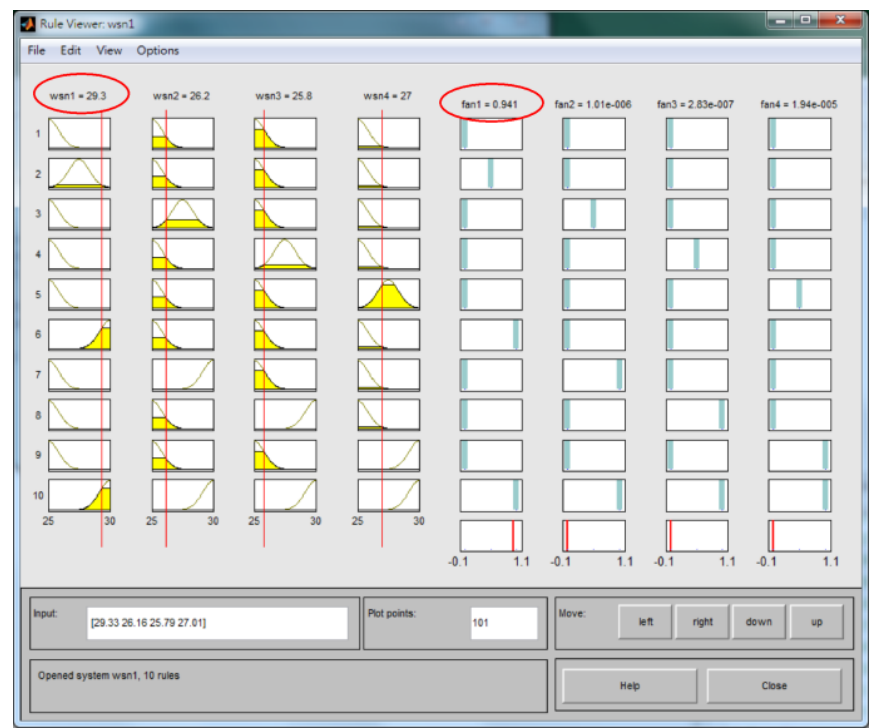

Figure 8 (b)

ANFIS inference result diagram

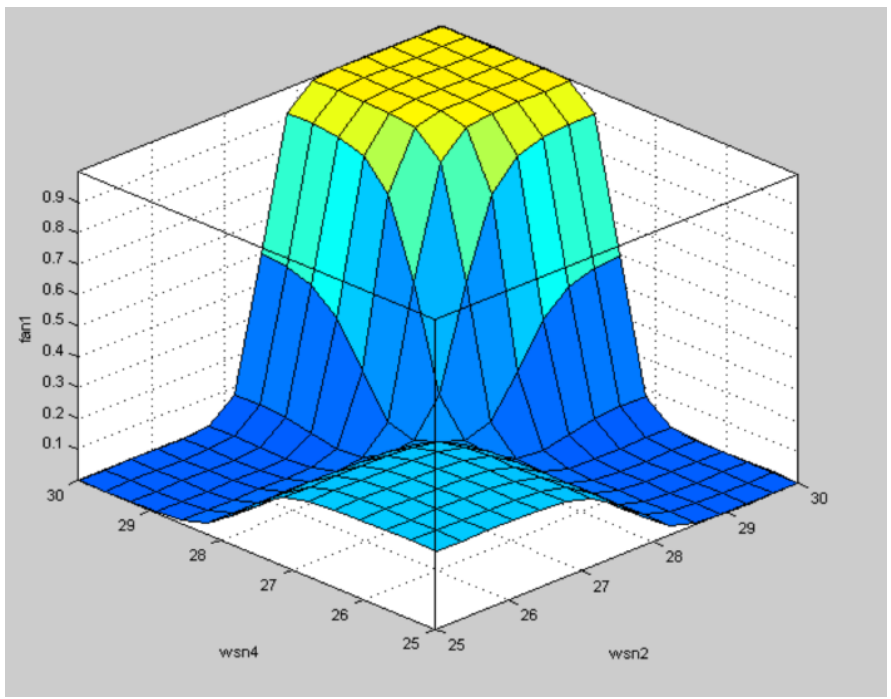

Figure 8 (c)

One of the ANFIS inference surface diagrams 

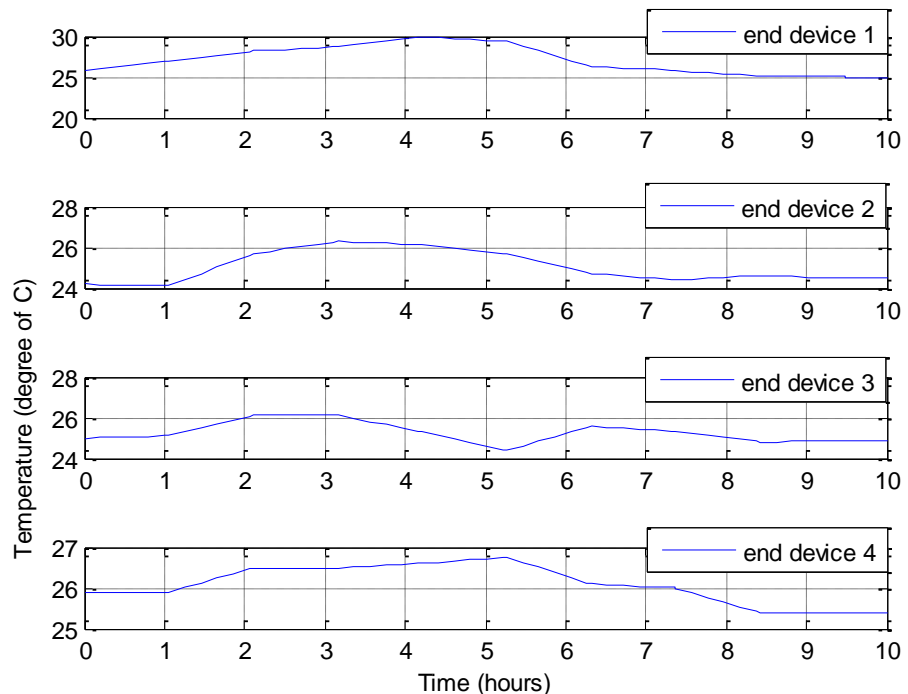

Figure 9 (a)

Diagram of measured temperatures of four corners indoor
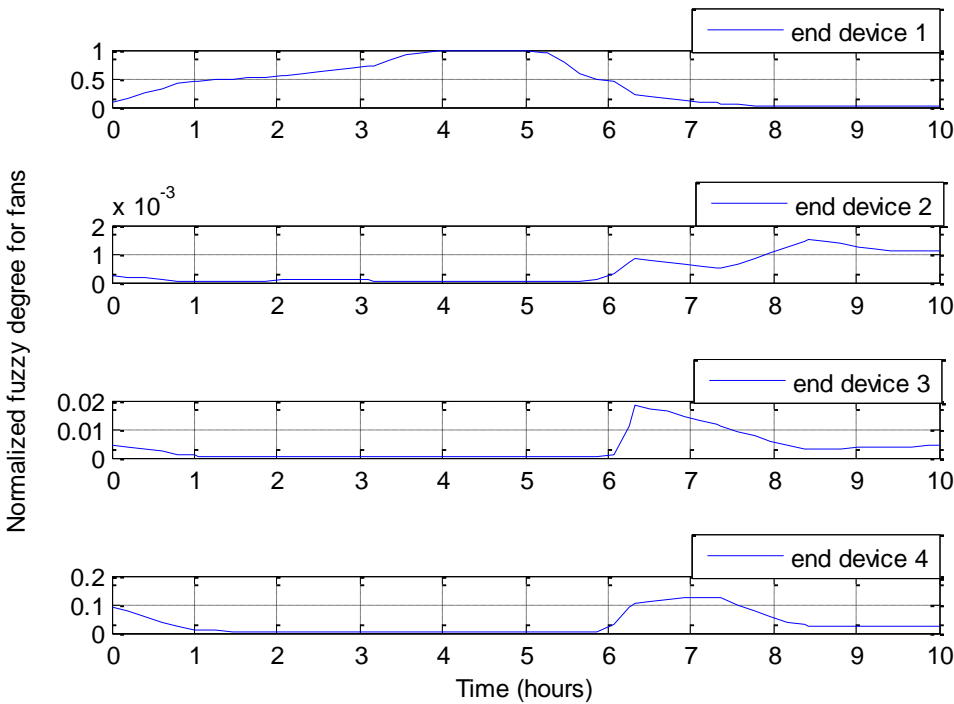

Figure 9 (b)

Diagram of simulation results of four corners indoor 


\section{Conclusions}

A design method for the control of indoor an air conditioner by using the ANFISbased WSN is proposed. This paper has successfully demonstrated the application of the WSN to monitor the indoor temperature. Physical verifications and simulations are also successfully demonstrated to show that satisfactory performance of the ANFIS-based WSN control of the motors of the fans, of the data collection and of temperature monitoring.

\section{Acknowledgments}

This paper is partially funded by teacher's research project of Taoyuan Innovation Institute of Technology.

\section{References}

[1] FT-625x Development Kits User Guide (Tradition Chinese version) 2012 (http://surewin.com.tw)

[2] R. Belbachir, Z. M. Mekkakia and A. Kies: Towards a New Approach in Available Bandwidth Measures on Mobile Ad Hoc Networks, Acta Polytechnica Hungarica, Vol. 8, 2011, pp. 133-148

[3] Gy. Mester: Intelligent Mobile Robot Motion Control in Unstructured Environments, Acta Polytechnica Hungarica, Vol. 7, 2010, pp. 153-165

[4] K. Romer and F. Mattern: The Design Space of Wireless Sensor Networks, IEEE Journal on Wireless Communications, Vol. 11, 2004, pp. 54-61

[5] L. X. Guo, Y. M. Zhang and L. P. Zhao: Motion Navigation and Fuzzy Control of Mobile Robots in Wireless Sensor Networks, Sensor Letters, Vol. 9, 2011, pp. 2000-2005

[6] V. C. Gungor and G. P. Hancke: Industrial Wireless Sensor Networks: Challenges, Design Principles, and Technical Approaches, IEEE Transactions on Industrial Electronics, Vol. 56, 2009, pp. 4258-4265

[7] H. B. Lee, L. J. Park, S. W. Park, T. Y. Chung and J. H. Moon: Interactive Remote Control of Legacy Home Appliances through a Virtually Wired Sensor Network, IEEE Transactions on Consumer Electronics, Vol. 56, 2010, pp. 2241-2248

[8] A. Schoofs, G. M. P. O'Hare and A. G. Ruzzelli: Debugging Low-Power and Lossy Wireless Networks: a Survey, IEEE Communications Surveys and Tutorials, Vol. 14, 2012, pp. 311-321

[9] H. A. Tanaka, H. Nakao and K. Shinohara: Self-Organizing Timing Allocation Mechanism in Distributed Wireless Sensor Networks, IEICE Electronics Express, Vol. 6, 2009, pp. 1562-1568 
[10] Y. J. Mon, C. M. Lin and I. J. Rudas: Wireless sensor Network (WSN) Control for Indoor Temperature Monitoring, Acta Polytechnica Hungarica, Vol. 9, No. 6, 2012, pp. 17-28

[11] J. S. R. Jang: ANFIS: Adaptive-Network-based Fuzzy Inference System, IEEE Transactions on Systems, Man and Cybernetics, Vol. 23, 1993, pp. 665-685

[12] Y. J. Mon: Airbag Controller Designed by Adaptive-Network-based Fuzzy Inference System (ANFIS), Fuzzy Sets and Systems, Vol. 158, 2007, pp. 2706-2714

[13] S. Kurnaz and O. Çetin: Autonomous Navigation and Landing Tasks for Fixed Wing Small Unmanned Aerial Vehicles, Acta Polytechnica Hungarica, Vol. 7, No. 1, 2010, pp. 87-102

[14] A. Azadeh, M. Saberi, V. Nadimi, M. Iman and A. Behrooznia: An Integrated Intelligent Neuro-Fuzzy Algorithm for Long-Term Electricity Consumption: Cases of Selected EU Countries, Acta Polytechnica Hungarica, Vol. 7, No. 4, 2010, pp. 71-90

[15] MATLAB ${ }^{\mathrm{TM}}$ fuzzy toolbox user guide (www.mathworks.com)

[16] Jennic Board API Reference Manual (JN-RM-2003), Jennic Inc., 2007 (www.jennic.com)

[17] Jennic 802.15.4 Stack API Reference Manual (JN-RM-2002), Jennic Inc., 2007 (www.jennic.com) 